Article

\title{
State Feedback with Memory for Constrained Switched Positive Linear Systems
}

\section{Jinjin Liu * and Kanjian Zhang}

Key Laboratory of Measurement and Control of CSE, Ministry of Education, School of Automation, Southeast University, Nanjing 210096, China; E-Mails: kjzhang@ seu.edu.cn

* Author to whom correspondence should be addressed; E-Mail: jjliu @ hotmail.com; Tel.: +86-25-8379-5609.

Academic Editor: J. A. Tenreiro Machado

Received: 4 February 2015 / Accepted: 23 April 2015 / Published: 27 April 2015

\begin{abstract}
In this paper, the stabilization problem in switched linear systems with time-varying delay under constrained state and control is investigated. The synthesis of bounded state-feedback controllers with memory ensures that a closed-loop state is positive and stable. Firstly, synthesis with a sign-restricted (nonnegative and negative) control is considered for general switched systems; then, the stabilization issue under bounded controls including the asymmetrically bounded controls and states constraints are addressed. In addition, the results are extended to systems with interval and polytopic uncertainties. All the proposed conditions are solvable in term of linear programming. Numerical examples illustrate the applicability of the results.
\end{abstract}

Keywords: switched linear systems; positive systems; bounded control; delay; linear programming

\section{Introduction}

Many systems in practice always involve kinds of constrained signals, such as the bind on the state and control input. Moreover, controllers are to be designed in order to guarantee the stability and some desired performance under the limitations of the system. Several approaches have been put forward to address this problem, such as positive invariance [1,2], predictive control [3] and the polynomial 
approach [4]. In addition, it is shown that linear programming is also powerful for solving controller synthesis in presence of bounded state and input, see $[5,6]$ and the references therein.

Switched linear systems, which consist of a family of subsystems and a switching signal orchestrates switching among these subsystems, have drawn a lot of attention in recent decades. There are several papers on the stability and stabilization issues of switched linear systems [7-12]. In [7,8], a sufficient and necessary condition was proposed for the existence of a common quadratic Lyapunov function under arbitrary switching. The multiple Lyapunov function method has been usually pursued in under average dwell time switching $[9,10]$. The switching stabilization problem of switched linear systems has been also studied in [11,12]. On the other hand, dynamic systems whose states are always nonnegative are coined as positive systems [13]. Positive switched systems consist of a family of positive subsystems and a switching law specifying when and how the switching takes place have attracted much attention due to their wide range of applications in network congestion control [14], formation flying [15], and medical treatment [16]. Due to some unpredictable factors, such as the changes of operation environment, the aging of the equipment, it is significant to research the stabilization problem of uncertain systems. Moreover, many real systems can be modeled as systems with interval and polytopic uncertainties.

Recently, stability and stabilization properties for switched positive linear systems have been studied by resorting to the linear copositive Lyapunov function approaches. For arbitrary switching, common linear copositive Lyapunov function is applied in [17-20]. Some other results about switched positive linear systems can refer to literature [21-26]. The stability and robust stabilization issues by the multiple linear Lyapunov function approach is considered under the dwell time switching in [21,22]. The mutually relations among the linear copositive functions, quadratic copositive functions, and the quadratic positive definite functions are gives in [23]. For systems with exogenous disturbance, the $L_{1}$-gain analysis and control synthesis are studied in [24,25]. The concept of finite-time stability to switched positive linear systems is extended by reference [26]. In practice, delay phenomena widely exist in dynamic systems $[27,28]$. Then the concept of stability to switched positive linear systems with delays is shown by [29]. The constrained control of positive linear systems with multiple delays is investigated in [30,31]. The system states to the nonnegative orthant by state feedback with memory and memoryless controller are derived in reference $[32,33]$. The robust stabilization problems under constrained controls for system with interval and polytopic uncertainties are investigated in reference [34]. For switched positive linear systems with multiple delays, the stability problem both continuous and discrete time systems are solved in [35]. The controller synthesis for witched positive linear systems with delays is a concern of [36]; specifically, a nonnegative memoryless controller subject to the nonnegative limitation on the system matrix. Finding a set of controllers in order to lift the limitation provides the motivation for the research presented in this paper.

In this paper, the stabilization problem for switched linear systems with time-varying delay is addressed by using the constrained state feedback controller with memory. The main contributions can be summarized as follows: (i) sufficient conditions are established for the existence of such controller under the sign constraint or nonsymmetrical bounds, which guarantees that the closed-loop systems are positive and asymptotically stable. (ii) the results are extended to the systems with polytopic and interval uncertainties. (iii) the approach we proposed not only can be applied to switched positive linear systems but also be true to general switched linear systems with delay. Thus, it can be interpreted as enforcing 
the system with delay to be positive by state-feedback with memory. The linear programming method is utilized to solve this controller synthesis issue with bounded controls. The rest of paper is organized as follows. In Section 2, preliminary results are provided. Section 3 addresses the controller synthesis with bounded state and control input. Section 4 solves the constraint controller synthesis for systems with interval uncertainties. The controller synthesis for systems with polytopic uncertainties is studied in Section 5. Numerical examples are shown in Section 6. Section 7 concludes the paper.

Notations: The notations $\mathcal{R}^{n}$ and $\mathcal{R}^{m \times n}$ denote the $n$-dimensional Euclidean space and the space of $m \times n$ matrices, respectively. $h_{i}$ is the $i$-th component of vector $h \in \mathcal{R}^{n}$, and $h \succeq 0(h \succ 0)$ means that $h_{i} \geq 0\left(h_{i}>0\right)$. Similarly, $a_{i j}$ stands for the $i$-th row $j$-th column of matrix $A \in \mathcal{R}^{m \times n}, A \succeq 0(A \succ 0)$ implies that $a_{i j} \geq 0\left(a_{i j}>0\right)$ for $i=1,2, \ldots, m$ and $j=1,2, \ldots, n$. $A$ is called a Metzler matrix if the off-diagonal entries of $A$ are nonnegative, i.e., $a_{i j} \geq 0$ for all $i \neq j$.

\section{Problem Formulation and Preliminaries}

Consider the following continuous-time switched linear systems

$$
\begin{aligned}
& \dot{x}(t)=A_{\sigma(t)} x(t)+A_{d \sigma(t)} x(t-d(t))+B_{\sigma(t)} u(t), \quad t \geq 0 \\
& x(t)=\varphi(t), \quad t \leq 0
\end{aligned}
$$

where $x(\cdot) \in \mathcal{R}^{n}$ is system state, $u(\cdot) \in \mathcal{R}^{n}$ is control input. $d(\cdot) \succeq 0$ and $\varphi(\cdot)$ denote delay and initial function, respectively. $\sigma(\cdot)$ is an arbitrary switching signal takes values in a finite set $\mathcal{P}=\{1,2, \ldots, N\}$. When $t \in\left[t_{p-1}, t_{p}\right]$, it can be interpreted as the $\sigma\left(t_{p}\right)$-th or $p$-th system is active, $A_{p}=\left[a_{p i j}\right] \in \mathcal{R}^{n \times n}$, $A_{d p}=\left[a_{d p i j}\right] \in \mathcal{R}^{n \times n}$ and $B_{p}=\left[b_{p i j}\right] \in \mathcal{R}^{n \times m}$ are subsystem matrices.

Our main objective in this paper is to design a set of state feedback controllers

$$
u(t)=F_{p} x(t)+F_{d p} x(t-d(t)),
$$

such that the closed-loop system given by

$$
\dot{x}(t)=\left(A_{p}+B_{p} F_{p}\right) x(t)+\left(A_{d p}+B_{p} F_{d p}\right) x(t-d(t)),
$$

is not only positive but also asymptotically stable under arbitrary switching.

In order to achieve the goal, some preliminary results are now given.

Definition 1. [36] System (1) is called positive if for any initial condition $\varphi(t) \succeq 0(t \leq 0)$ and $u(t) \succeq$ $0(t \geq 0)$, the corresponding trajectory $x(t) \succeq 0(t \geq 0)$ holds for arbitrary switching signal.

Lemma 1. [29] The autonomous system of $(1)(u(t) \equiv 0)$ is positive if and only if $A_{p}$ is a Metzler matrix and $A_{d p} \succeq 0$.

Clearly, the closed-loop system (3) is positive if $\left(A_{p}+B_{p} F_{p}\right)$ is a Metzler matrix and $\left(A_{d p}+B_{p} F_{d p}\right) \succeq$ 0 for each $p \in \mathcal{P}$.

Lemma 2. [29] Assume that time delay system (1) is positive, then its unforced system $(u(t)=0)$ is asymptotically stable for any switching signals if there exists a vector $h \succ 0$ in $\mathcal{R}^{n}$ such that $\left(A_{p}+A_{d p}\right) h \prec 0$ for each $p \in \mathcal{P}$. 
Lemma 3. [36] Consider the autonomous systems of (1), then for a given vector $h \succ 0$, we have $0 \preceq x(t) \preceq h$ for any initial condition satisfying $0 \preceq \varphi(t) \preceq h$ if and only if $A_{p}$ are Metzler matrices, $A_{d p} \succeq 0$ and $\left(A_{p}+A_{d p}\right) h \preceq 0$ for $p \in \mathcal{P}$.

\section{Synthesis with Bounded Control}

In this section, we consider the stabilization problem for switched linear systems with delay via bounded state feedback controllers with memory under arbitrary switching signal.

\subsection{Sign-Restricted Control}

This subsection solve stabilization issue of system by nonnegative and negative controls.

Theorem 1. Consider system (1), if there exist matrices $H=\operatorname{diag}\left\{h_{1}, h_{2}, \ldots, h_{n}\right\} \succ 0, Y_{p}=\left[y_{p i j}\right] \succeq$ $0 \in \mathcal{R}^{m \times n}$ and $Y_{d p}=\left[y_{d p i j}\right] \succeq 0 \in \mathcal{R}^{m \times n}$ for $p \in \mathcal{P}$ such that

$$
\begin{aligned}
a_{p i j} h_{j}+\sum_{k=1}^{m} b_{p i k} y_{p k j} & \geq 0, \text { for } \forall 1 \leq i \neq j \leq n, \\
a_{d p i j} h_{j}+\sum_{k=1}^{m} b_{p i k} y_{d p k j} & \geq 0, \text { for } \forall 1 \leq i, j \leq n, \\
\sum_{j=1}^{n}\left(a_{p i j}+a_{d p i j}\right) h_{j}+\sum_{j=1}^{n} \sum_{k=1}^{m} b_{p i k}\left(y_{p k j}+y_{d p k j}\right) & <0, \text { for } \forall 1 \leq i \leq n,
\end{aligned}
$$

then the closed-loop system (3) is positive and stable by a set of nonnegative controllers $u(t)=$ $F_{\sigma(t)} x(t)+F_{d \sigma(t)} x(t-d(t))$ under arbitrary switching signal. For $p \in \mathcal{P}, F_{p}$ and $F_{d p}$ are given by

$$
F_{p}=Y_{p} H^{-1}, F_{d p}=Y_{d p} H^{-1} .
$$

Proof. Firstly, it follows from (7) that $y_{p k j}=f_{p k j} h_{j}$, then

$$
a_{p i j} h_{j}+\sum_{k=1}^{m} b_{p i k} f_{p k j} h_{j}=a_{p i j} h_{j}+\sum_{k=1}^{m} b_{p i k} y_{p k j} .
$$

By virtue of (4) together with that $h_{j} \geq 0$, we can obtain that the off-diagonal components of $A_{p}+B_{p} F_{p}$ are nonnegative, or $A_{p}+B_{p} F_{p}$ is a Metzler matrix. Similarly we can get $A_{d p}+B_{p} F_{d p} \succeq 0$ from inequality (5). So the closed-loop system (3) is positive.

Secondly, consider the element of the system matrices and manipulate them according to the certain way

$$
\begin{aligned}
{\left[\left(A_{p}+B_{p} F_{p}+A_{d p}+B_{p} F_{d p}\right) h\right]_{i} } & =\sum_{j=1}^{n}\left[\left(a_{p i j}+a_{d p i j}+\sum_{k=1}^{m} b_{p i k} f_{p k j}+\sum_{k=1}^{m} b_{p i k} f_{d p k j}\right) h_{j}\right] \\
& =\sum_{j=1}^{n}\left(a_{p i j}+a_{d p i j}\right) h_{j}+\sum_{j=1}^{n} \sum_{k=1}^{m} b_{p i k}\left(y_{p k j}+y_{d p k j}\right) .
\end{aligned}
$$

Note that (6), we have $\left(A_{p}+B_{p} F_{p}+A_{d p}+B_{p} F_{d p}\right) h \prec 0$. Therefore, the closed-loop system (3) is asymptotically stable via Lemma 2 .

Finally, $F_{p} \succeq 0$ and $F_{d p} \succeq 0$ are established from $H \succ 0, Y_{p} \succeq 0$ and $Y_{d p} \succeq 0$. Thus, the state feedback controller with memory is nonnegative. 
Remark 1. Observe that linear programming (LP) conditions in Theorem 1 do not impose any restriction on the original system, i.e., $A_{p}$ is not necessarily a Metzler matrix and $A_{d p}$ is not necessarily positive matrix, so this can be viewed as a controlled positivity problem. Certainly, the results of Theorem 1 can be also applied to switched positive linear systems. Finally, negative results can derived similarly, that is Corollary 1 in the following.

Corollary 1. Consider system (1), if there exist matrices $H=\operatorname{diag}\left\{h_{1}, h_{2}, \ldots, h_{n}\right\} \succ 0, Y_{p}=\left[y_{p i j}\right] \prec$ $0 \in \mathcal{R}^{m \times n}$ and $Y_{d p}=\left[y_{d p i j}\right] \prec 0 \in \mathcal{R}^{m \times n}$ for $p \in \mathcal{P}$ such that

$$
\begin{aligned}
a_{p i j} h_{j}+\sum_{k=1}^{m} b_{p i k} y_{p k j} & \geq 0, \text { for } \forall 1 \leq i \neq j \leq n, \\
a_{d p i j} h_{j}+\sum_{k=1}^{m} b_{p i k} y_{d p k j} & \geq 0, \text { for } \forall 1 \leq i, j \leq n, \\
\sum_{j=1}^{n}\left(a_{p i j}+a_{d p i j}\right) h_{j}+\sum_{j=1}^{n} \sum_{k=1}^{m} b_{p i k}\left(y_{p k j}+y_{d p k j}\right) & <0, \text { for } \forall 1 \leq i \leq n,
\end{aligned}
$$

then the closed-loop system (3) is positive and stable by a set of negative controllers $u(t)=F_{\sigma(t)} x(t)+$ $F_{d \sigma(t)} x(t-d(t))$ for arbitrary switching signal. For $p \in \mathcal{P}, F_{p}$ and $F_{d p}$ are given by

$$
F_{p}=Y_{p} H^{-1}, F_{d p}=Y_{d p} H^{-1}
$$

\subsection{Bounded and Sign-Restricted Controls}

The synthesis issue for switched linear systems in present of bounded and sign-restricted controls is solved in this subsection.

Next, we focused on stabilization of switched linear systems by bounded nonnegative control, that is $0 \preceq u(t) \preceq \bar{u}$. For fixed $\bar{u} \succ 0$, find $h \succ 0$ corresponding to bounded control law subject to the resulting closed-loop system is positive and asymptotically stable for $0 \preceq \varphi(t) \preceq h$.

Theorem 2. Consider system (1) with nonnegative bounded control, if there exist matrices $H=$ $\operatorname{diag}\left\{h_{1}, h_{2}, \ldots, h_{n}\right\} \succ 0, Y_{p}=\left[y_{p i j}\right] \succeq 0 \in \mathcal{R}^{m \times n}$ and $Y_{d p}=\left[y_{d p i j}\right] \succeq 0 \in \mathcal{R}^{m \times n} p \in \mathcal{P}$ satisfying

$$
\begin{aligned}
a_{p i j} h_{j}+\sum_{k=1}^{m} b_{p i k} y_{p k j} & \geq 0, \text { for } \forall 1 \leq i \neq j \leq n, \\
a_{d p i j} h_{j}+\sum_{k=1}^{m} b_{p i k} y_{d p k j} & \geq 0, \text { for } \forall 1 \leq i, j \leq n, \\
\sum_{j=1}^{n}\left(a_{p i j}+a_{d p i j}\right) h_{j}+\sum_{j=1}^{n} \sum_{k=1}^{m} b_{p i k}\left(y_{p k j}+y_{d p k j}\right) & <0, \text { for } \forall 1 \leq i \leq n, \\
\sum_{j=1}^{n}\left(y_{p k j}+y_{d p k j}\right) & \leq \bar{u}_{k}, \text { for } \forall 1 \leq k \leq m,
\end{aligned}
$$


then the closed-loop system (3) is not only positive but also asymptotically stable by a set of controllers $u(t)=F_{\sigma(t)} x(t)+F_{d \sigma(t)} x(t-d(t))$ for arbitrary switching signal. For $p \in \mathcal{P}, F_{p}$ and $F_{d p}$ can be derived by

$$
F_{p}=Y_{p} H^{-1}, F_{d p}=Y_{d p} H^{-1} .
$$

Moreover, define $\bar{u}=\left[\bar{u}_{1}, \bar{u}_{2}, \cdots, \bar{u}_{m}\right]^{T}$, then $0 \preceq u(t) \preceq \bar{u}$ is established for any initial condition satisfying $0 \preceq x(0) \preceq h$.

Proof. We can prove the closed-loop system (3) is positive and asymptotically stable similar to Theorem 1 . Then by Lemma $3,0 \preceq x(t) \preceq h$ holds on for any initial condition satisfying $0 \preceq \varphi(t) \preceq h$. Combining with inequality (17), it is easy to see that the state-feedback control with memory satisfies $0 \preceq u(t) \preceq \bar{u}$.

In this sequel, consider the controller synthesis of switched linear systems by bounded and negative control. For prescribed $\tilde{u} \prec 0$, our objective is to find $h \succ 0$ corresponding to the bounded control $\tilde{u} \preceq u(t) \preceq 0$ subject to the resulting closed-loop system is positive and asymptotically stable for $0 \preceq \varphi(t) \preceq h$.

Corollary 2. Consider system (1), if there exist matrices $H=\operatorname{diag}\left\{h_{1}, h_{2}, \ldots, h_{n}\right\} \succ 0, Y_{p}=\left[y_{p i j}\right] \prec$ $0 \in \mathcal{R}^{m \times n}$ and $Y_{d p}=\left[y_{d p i j}\right] \prec 0 \in \mathcal{R}^{m \times n}$ for $p \in \mathcal{P}$ satisfying

$$
\begin{aligned}
a_{p i j} h_{j}+\sum_{k=1}^{m} b_{p i k} y_{p k j} \geq 0, \text { for } \forall 1 \leq i \neq j \leq n, \\
a_{d p i j} h_{j}+\sum_{k=1}^{m} b_{p i k} y_{d p k j} \geq 0, \text { for } \forall 1 \leq i, j \leq n, \\
\sum_{j=1}^{n}\left(a_{p i j}+a_{d p i j}\right) h_{j}+\sum_{j=1}^{n} \sum_{k=1}^{m} b_{p i k}\left(y_{p k j}+y_{d p k j}\right)<0, \text { for } \forall 1 \leq i \leq n, \\
\tilde{u}_{k} \leq \sum_{j=1}^{n}\left(y_{p k j}+y_{d p k j}\right), \quad \text { for } \forall 1 \leq k \leq m,
\end{aligned}
$$

then the closed-loop system (3) is positive and asymptotically stable under a set of controllers $u(t)=$ $F_{\sigma(t)} x(t)+F_{d \sigma(t)} x(t-d(t))$ for arbitrary switching signal. For $p \in \mathcal{P}, F_{p}$ and $F_{d p}$ can be obtained by

$$
F_{p}=Y_{p} H^{-1}, F_{d p}=Y_{d p} H^{-1}
$$

Moreover, define $\tilde{u}=\left[\tilde{u}_{1}, \tilde{u}_{2}, \cdots, \tilde{u}_{m}\right]^{T}$, then $\tilde{u} \preceq u(t) \preceq 0$ is established for any initial condition satisfying $0 \preceq x(0) \preceq h$.

\subsection{Asymmetrically Bounded Control}

This subsection considers the following problem: for prescribed $\bar{u}^{1} \succ 0$ and $\bar{u}^{2} \succ 0$, the aim is to determine bounded control law $-\bar{u}^{1} \preceq u(t) \preceq \bar{u}^{2}$ and vector $h \succ 0$ such that the resulting closed-loop system is positive and asymptotically stable for any initial condition $0 \preceq \varphi(t) \preceq h$. 
Theorem 3. Consider system (1), if there exist matrices $H=\operatorname{diag}\left\{h_{1}, h_{2}, \ldots, h_{n}\right\} \succ 0$ and $Y_{p}^{l}=$ $\left[y_{p i j}^{l}\right] \succeq 0 \in \mathcal{R}^{m \times n}, Y_{d p}^{l}=\left[y_{d p i j}^{l}\right] \succeq 0 \in \mathcal{R}^{m \times n} p \in \mathcal{P}, l \in\{1,2$,$\} subject to$

$$
\begin{aligned}
a_{p i j} h_{j}+\sum_{k=1}^{m} b_{p i k}\left(y_{p k j}^{1}-y_{p k j}^{2}\right) & \geq 0, \text { for } \forall 1 \leq i \neq j \leq n, \\
a_{d p i j} h_{j}+\sum_{k=1}^{m} b_{p i k}\left(y_{d p k j}^{1}-y_{d p k j}^{2}\right) & \geq 0, \text { for } \forall 1 \leq i, j \leq n,
\end{aligned}
$$

$\sum_{j=1}^{n}\left(a_{p i j}+a_{d p i j}\right) h_{j}+\sum_{j=1}^{n} \sum_{k=1}^{m} b_{p i k}\left(y_{p k j}^{1}+y_{d p k j}^{1}-y_{l p k j}^{2}-y_{d p k j}^{2}\right)<0$, for $\forall 1 \leq i \leq n$,

$$
\begin{aligned}
& \sum_{j=1}^{n}\left(y_{p k j}^{1}+y_{d p k j}^{1}\right) \leq \bar{u}_{k}^{2}, \text { for } \forall 1 \leq k \leq m, \\
& \sum_{j=1}^{n}\left(y_{p k j}^{2}+y_{d p k j}^{2}\right) \leq \bar{u}_{k}^{1}, \text { for } \forall 1 \leq k \leq m,
\end{aligned}
$$

then the closed-loop system (3) is not only positive but also asymptotically stable by a set of controllers with memory $u(t)=F_{\sigma(t)} x(t)+F_{d \sigma(t)} x(t-d(t))$. For $p \in \mathcal{P}, F_{p} F_{d p}$ can be computed as follows

$$
F_{p}=\left(Y_{p}^{1}-Y_{p}^{2}\right) H^{-1}, F_{d p}=\left(Y_{d p}^{1}-Y_{d p}^{2}\right) H^{-1} .
$$

Furthermore, let $\bar{u}^{1}=\left[\bar{u}_{1}^{1}, \bar{u}_{2}^{1}, \cdots, \bar{u}_{m}^{1}\right]^{T}$ and $\bar{u}^{2}=\left[\bar{u}_{1}^{2}, \bar{u}_{2}^{2}, \cdots, \bar{u}_{m}^{2}\right]^{T}$, then $-\bar{u}^{1} \preceq u(t) \preceq \bar{u}^{2}$ sets up for any initial condition fulfilling $0 \preceq \varphi(t) \preceq h$.

Proof. It follows the proof of Theorem 1 because that gain matrix $F_{p}$ can be described as: $F_{p}=F_{p}^{1}-F_{p}^{2}$, where $F_{p}^{1}=Y_{p}^{1} H^{-1}$ and $F_{p}^{2}=Y_{p}^{2} H^{-1}$.

\subsection{Synthesis with State Constraints}

This subsection concerned with stabilization problem with state constraints: for given $\bar{x} \succ 0$ choose controller with memory together with initial condition $0 \preceq \varphi(t) \preceq h$ such that $0 \preceq x \preceq \bar{x}$ sets up, the resulting closed-loop system is positive and asymptotically stable.

Theorem 4. Consider system (1), if there exist matrices $H=\operatorname{diag}\left\{h_{1}, h_{2}, \ldots, h_{n}\right\} \succ 0, Y_{p}=\left[y_{p i j}\right] \in$ $\mathcal{R}^{m \times n}$ and $Y_{d p}=\left[y_{d p i j}\right] \in \mathcal{R}^{m \times n} p \in \mathcal{P}$ such that

$$
\begin{aligned}
a_{p i j} h_{j}+\sum_{k=1}^{m} b_{p i k} y_{p k j} & \geq 0, \text { for } \forall 1 \leq i \neq j \leq n, \\
a_{d p i j} h_{j}+\sum_{k=1}^{m} b_{p i k} y_{d p k j} & \geq 0, \text { for } \forall 1 \leq i, j \leq n, \\
\sum_{j=1}^{n}\left(a_{p i j}+a_{d p i j}\right) h_{j}+\sum_{j=1}^{n} \sum_{k=1}^{m} b_{p i k}\left(y_{p k j}+y_{d p k j}\right) & <0, \text { for } \forall 1 \leq i \leq n, \\
h_{j} & \leq \bar{x}_{j}, \text { for } \forall 1 \leq j \leq n,
\end{aligned}
$$


then the closed-loop system (3) is not only positive but also asymptotically stable by a set of controllers $u(t)=F_{\sigma(t)} x(t)+F_{d \sigma(t)} x(t-d(t))$ for arbitrary switching signal. For $p \in \mathcal{P}, F_{p}$ and $F_{d p}$ are given by

$$
F_{p}=Y_{p} H^{-1}, F_{d p}=Y_{d p} H^{-1} .
$$

Furthermore, let $\bar{x}=\left[\bar{x}_{1}, \bar{x}_{2}, \cdots, \bar{x}_{n}\right]^{T}$, then $0 \preceq x(t) \preceq \bar{x}$ holds on for $0 \preceq \varphi(t) \preceq h$.

Proof. According to the proof of Theorem 1, it is easy to finish, so it is omitted.

\section{Synthesis with Interval Uncertainties}

This section handles the robust stabilization issue via bounded state feedback for systems which are composed of interval uncertain subsystems. In the following, we consider the synthesis via bounded control, that is an extension of the result of Section 3. Assume that the subsystem matrices are unknown and bounded as follows:

$$
\underline{A}_{p} \preceq A_{p} \preceq \bar{A}_{p}, \quad \underline{A}_{d p} \preceq A_{d p} \preceq \bar{A}_{d p}, \quad \underline{B}_{p} \preceq B_{p} \preceq \bar{B}_{p}
$$

\subsection{Sign-Restricted Control}

An approach for solving the robust stabilization issue of systems with interval uncertainties by nonnegative and negative controls is presented in this subsection.

Theorem 5. Consider system (1) with interval uncertainties, if there exist matrices $H=$ $\operatorname{diag}\left\{h_{1}, h_{2}, \ldots, h_{n}\right\} \succ 0, Y_{p}=\left[y_{p i j}\right] \succeq 0 \in \mathcal{R}^{m \times n}$ and $Y_{d p}=\left[y_{\text {dpij }}\right] \succeq 0 \in \mathcal{R}^{m \times n}$ for $p \in \mathcal{P}$ satisfying

$$
\begin{aligned}
\underline{a}_{p i j} h_{j}+\sum_{k=1}^{m} \underline{b}_{p i k} y_{p k j} & \geq 0, \text { for } \forall 1 \leq i \neq j \leq n, \\
\underline{a}_{d p i j} h_{j}+\sum_{k=1}^{m} \underline{b}_{p i k} y_{d p k j} & \geq 0, \text { for } \forall 1 \leq i, j \leq n, \\
\sum_{j=1}^{n}\left(\bar{a}_{p i j}+\bar{a}_{d p i j}\right) h_{j}+\sum_{j=1}^{n} \sum_{k=1}^{m} \bar{b}_{p i k}\left(y_{p k j}+y_{d p k j}\right) & <0, \text { for } \forall 1 \leq i \leq n,
\end{aligned}
$$

then the closed-loop system (3) is positive and stable by a set of nonnegative controllers $u(t)=$ $F_{\sigma(t)} x(t)+F_{d \sigma(t)} x(t-d(t))$ for arbitrary switching signal. For $p \in \mathcal{P}, F_{p}$ and $F_{d p}$ are given by

$$
F_{p}=Y_{p} H^{-1}, F_{d p}=Y_{d p} H^{-1}
$$

Proof. From (36) together with the fact that $A_{p}+B_{p} F_{p} \succeq \underline{A}_{p}+\underline{B}_{p} F_{p}$, then $A_{p}+B_{p} F_{p}$ is a Metzler matrix. Similarly we can get $A_{d p}+B_{p} F_{d p} \succeq 0$ from inequality (37). So the closed-loop system is positive. Next, note that (38), we have that $\left(A_{p}+B_{p} F_{p}+A_{d p}+B_{p} F_{d p}\right) h \preceq\left(\bar{A}_{p}+\bar{B}_{p} F_{p}+\bar{A}_{d p}+\bar{B}_{p} F_{d p}\right) h \prec 0$. Therefore, the closed-loop system (3) with uncertainties is asymptotically stable by Lemma 2.

Finally, $F_{p} \succeq 0$ and $F_{d p} \succeq 0$ are established from $H \succ 0, Y_{p} \succeq 0$ and $Y_{d p} \succeq 0$. Thus, the state feedback controller with memory is nonnegative. 
Corollary 3. Consider system (1) with interval uncertainties, if there exist matrices $H=$ $\operatorname{diag}\left\{h_{1}, h_{2}, \ldots, h_{n}\right\} \succ 0, Y_{p}=\left[y_{p i j}\right] \prec 0 \in \mathcal{R}^{m \times n}$ and $Y_{d p}=\left[y_{\text {dpij }}\right] \prec 0 \in \mathcal{R}^{m \times n}$ for $p \in \mathcal{P}$ such that

$$
\begin{aligned}
\underline{a}_{p i j} h_{j}+\sum_{k=1}^{m} \bar{b}_{p i k} y_{p k j} & \geq 0, \text { for } \forall 1 \leq i \neq j \leq n, \\
\underline{a}_{d p i j} h_{j}+\sum_{k=1}^{m} \bar{b}_{p i k} y_{d p k j} & \geq 0, \text { for } \forall 1 \leq i, j \leq n, \\
\sum_{j=1}^{n}\left(\bar{a}_{p i j}+\bar{a}_{d p i j}\right) h_{j}+\sum_{j=1}^{n} \sum_{k=1}^{m} \underline{b}_{p i k}\left(y_{p k j}+y_{d p k j}\right) & <0, \text { for } \forall 1 \leq i \leq n,
\end{aligned}
$$

then the closed-loop system (3) is not only positive but also stable by a set of negative controllers $u(t)=$ $F_{\sigma(t)} x(t)+F_{d \sigma(t)} x(t-d(t))$ for arbitrary switching signal. For $p \in \mathcal{P}, F_{p}$ and $F_{d p}$ can be computed as follows

$$
F_{p}=Y_{p} H^{-1}, F_{d p}=Y_{d p} H^{-1}
$$

\subsection{Bounded and Sign-Restricted Controls}

This part considers the controller synthesis issue under sign-restricted and bounded controls for system with interval uncertainties. The aim here is to design the constrained controller such that the closed-loop system is positive and asymptotically stable.

Theorem 6. Consider system (1) with interval uncertainties, if there exist matrices $H=$ $\operatorname{diag}\left\{h_{1}, h_{2}, \ldots, h_{n}\right\} \succ 0, Y_{p}=\left[y_{p i j}\right] \succeq 0 \in \mathcal{R}^{m \times n}$ and $Y_{d p}=\left[y_{d p i j}\right] \succeq 0 \in \mathcal{R}^{m \times n} p \in \mathcal{P}$ such that

$$
\begin{aligned}
\underline{a}_{p i j} h_{j}+\sum_{k=1}^{m} \underline{b}_{p i k} y_{p k j} & \geq 0, \text { for } \forall 1 \leq i \neq j \leq n, \\
\underline{a}_{d p i j} h_{j}+\sum_{k=1}^{m} \underline{b}_{p i k} y_{d p k j} & \geq 0, \text { for } \forall 1 \leq i, j \leq n, \\
\sum_{j=1}^{n}\left(\bar{a}_{p i j}+\bar{a}_{d p i j}\right) h_{j}+\sum_{j=1}^{n} \sum_{k=1}^{m} \bar{b}_{p i k}\left(y_{p k j}+y_{d p k j}\right) & <0, \text { for } \forall 1 \leq i \leq n, \\
\sum_{j=1}^{n}\left(y_{p k j}+y_{d p k j}\right) & \leq \bar{u}_{k}, \text { for } \forall 1 \leq k \leq m,
\end{aligned}
$$

then the closed-loop system (3) is not only positive but also asymptotically stable by a set of controllers $u(t)=F_{\sigma(t)} x(t)+F_{d \sigma(t)} x(t-d(t))$ for arbitrary switching signal. For $p \in \mathcal{P}, F_{p}$ and $F_{d p}$ are given by

$$
F_{p}=Y_{p} H^{-1}, F_{d p}=Y_{d p} H^{-1} .
$$

Besides, for fixed $\bar{u}=\left[\bar{u}_{1}, \bar{u}_{2}, \cdots, \bar{u}_{n}\right]^{T} \succ 0,0 \preceq u(t) \preceq \bar{u}$ is established for any initial condition satisfying $0 \preceq x(0) \preceq h$.

Proof. We can obtain the closed-loop system (3) is positive and asymptotically stable similarly with Theorem 5. Moreover, Combining with inequality (47), it is easy to see that the state-feedback control with memory satisfy $0 \preceq u(t) \preceq \bar{u}$. 
Corollary 4. Consider system (1) with interval uncertainties, if there exist matrices $H=$ $\operatorname{diag}\left\{h_{1}, h_{2}, \ldots, h_{n}\right\} \succ 0, Y_{p}=\left[y_{p i j}\right] \prec 0 \in \mathcal{R}^{m \times n}$ and $Y_{d p}=\left[y_{\text {dpij }}\right] \prec 0 \in \mathcal{R}^{m \times n}$ for $p \in \mathcal{P}$ subject to

$$
\begin{aligned}
& \underline{a}_{p i j} h_{j}+\sum_{k=1}^{m} \bar{b}_{p i k} y_{p k j} \geq 0, \text { for } \forall 1 \leq i \neq j \leq n, \\
& \underline{a}_{d p i j} h_{j}+\sum_{k=1}^{m} \bar{b}_{p i k} y_{d p k j} \geq 0, \text { for } \forall 1 \leq i, j \leq n, \\
& \sum_{j=1}^{n}\left(\bar{a}_{p i j}+\bar{a}_{d p i j}\right) h_{j}+\sum_{j=1}^{n} \sum_{k=1}^{m} \underline{b}_{p i k}\left(y_{p k j}+y_{d p k j}\right)<0, \text { for } \forall 1 \leq i \leq n, \\
& \tilde{u}_{k} \leq \sum_{j=1}^{n}\left(y_{p k j}+y_{d p k j}\right), \text { for } \forall 1 \leq k \leq m,
\end{aligned}
$$

then the closed-loop system (3) is not only positive but also asymptotically stable by a set of controllers $u(t)=F_{\sigma(t)} x(t)+F_{d \sigma(t)} x(t-d(t))$ for arbitrary switching signal. For $p \in \mathcal{P}, F_{p}$ and $F_{d p}$ are given by

$$
F_{p}=Y_{p} H^{-1}, F_{d p}=Y_{d p} H^{-1} .
$$

Moreover, choose $\tilde{u}=\left[\tilde{u}_{1}, \tilde{u}_{2}, \cdots, \tilde{u}_{m}\right]^{T} \prec 0$, then $\tilde{u} \preceq u(t) \preceq 0$ is established for any initial condition satisfying $0 \preceq x(0) \preceq h$.

\subsection{Asymmetrically Bounded Control}

The aim in this subsection is to address the following problems: for fixed $\bar{u}^{1} \succ 0$ and $\bar{u}^{2} \succ 0$, design control law $-\bar{u}^{1} \preceq u(t) \preceq \bar{u}^{2}$ such that the resulting closed-loop system is positive and asymptotically stable for any initial condition $0 \preceq \varphi(t) \preceq h$.

Theorem 7. Consider system (1) with interval uncertainties, if there exist matrices $H=$ $\operatorname{diag}\left\{h_{1}, h_{2}, \ldots, h_{n}\right\} \succ 0$ and $Y_{p}^{l}=\left[y_{p i j}^{l}\right] \succeq 0 \in \mathcal{R}^{m \times n}, Y_{d p}^{l}=\left[y_{d p i j}^{l}\right] \succeq 0 \in \mathcal{R}^{m \times n} p \in \mathcal{P}, l \in\{1,2$, such that

$$
\begin{aligned}
\underline{a}_{p i j} h_{j}+\sum_{k=1}^{m} \min \left\{\underline{b}_{p i k}\left(y_{p k j}^{1}-y_{p k j}^{2}\right), \bar{b}_{p i k}\left(y_{p k j}^{1}-y_{p k j}^{2}\right)\right\} & \geq 0, \text { for } \forall 1 \leq i \neq j \leq r(54) \\
\underline{a}_{d p i j} h_{j}+\sum_{k=1}^{m} \min \left\{\underline{b}_{p i k}\left(y_{d p k j}^{1}-y_{d p k j}^{2}\right), \bar{b}_{p i k}\left(y_{d p k j}^{1}-y_{d p k j}^{2}\right)\right\} & \geq 0, \text { for } \forall 1 \leq i, j \leq n,(55) \\
\sum_{j=1}^{n}\left(\bar{a}_{p i j}+\bar{a}_{d p i j}\right) h_{j}+\sum_{j=1}^{n} \sum_{k=1}^{m} \max \left\{\underline{b}_{p i k}\left(y_{p k j}^{1}+y_{d p k j}^{1}-y_{l p k j}^{2}-y_{d p k j}^{2}\right),\right. & \\
\left.\bar{b}_{p i k}\left(y_{p k j}^{1}+y_{d p k j}^{1}-y_{l p k j}^{2}-y_{d p k j}^{2}\right)\right\} & <0, \text { for } \forall 1 \leq i \leq n, \quad \text { (56) } \\
\sum_{j=1}^{n}\left(y_{p k j}^{1}+y_{d p k j}^{1}\right) & \leq \bar{u}_{k}^{2}, \text { for } \forall 1 \leq k \leq m,(57) \\
\sum_{j=1}^{n}\left(y_{p k j}^{2}+y_{d p k j}^{2}\right) & \leq \bar{u}_{k}^{1}, \text { for } \forall 1 \leq k \leq m,(58)
\end{aligned}
$$


then the closed-loop system (3) is not only positive but also asymptotically stable by a set of controllers with memory $u(t)=F_{\sigma(t)} x(t)+F_{d \sigma(t)} x(t-d(t))$. For $p \in \mathcal{P}, F_{p} F_{d p}$ are given by

$$
F_{p}=\left(Y_{p}^{1}-Y_{p}^{2}\right) H^{-1}, F_{d p}=\left(Y_{d p}^{1}-Y_{d p}^{2}\right) H^{-1} .
$$

Furthermore, take $\bar{u}^{1}=\left[\bar{u}_{1}^{1}, \bar{u}_{2}^{1}, \cdots, \bar{u}_{m}^{1}\right]^{T}$ and $\bar{u}^{2}=\left[\bar{u}_{1}^{2}, \bar{u}_{2}^{2}, \cdots, \bar{u}_{m}^{2}\right]^{T}$, then $-\bar{u}^{1} \preceq u(t) \preceq \bar{u}^{2}$ sets up for any initial condition fulfilling $0 \preceq \varphi(t) \preceq h$.

Proof. It follows the proof of Theorem 5 because that gain matrix $F_{p}$ can be described as: $F_{p}=F_{p}^{1}-F_{p}^{2}$, where $F_{p}^{1}=Y_{p}^{1} H^{-1}$ and $F_{p}^{2}=Y_{p}^{2} H^{-1}$.

\subsection{Synthesis with State Constraints}

This subsection considers synthesis problem for systems with interval uncertainties under state constraints: for fixed $\bar{x} \succ 0$, our aim is to design controller $u(k)$, such that the closed-loop system is asymptotically stable and state is constrained in the box: $0 \preceq x \preceq \bar{x}$.

Theorem 8. Consider system (1) with interval uncertainties, if there exist matrices $H=$ $\operatorname{diag}\left\{h_{1}, h_{2}, \ldots, h_{n}\right\} \succ 0, Y_{p}=\left[y_{p i j}\right] \in \mathcal{R}^{m \times n}$ and $Y_{d p}=\left[y_{\text {dpij }}\right] \in \mathcal{R}^{m \times n} p \in \mathcal{P}$ subject to

$$
\begin{aligned}
\underline{a}_{p i j} h_{j}+\sum_{k=1}^{m} \min \left\{\underline{b}_{p i k} y_{p k j}, \bar{b}_{p i k} y_{p k j}\right\} & \geq 0, \text { for } \forall 1 \leq i \neq j \leq n, \\
\underline{a}_{d p i j} h_{j}+\sum_{k=1}^{m} \min \left\{\underline{b}_{p i k} y_{d p k j}, \bar{b}_{p i k} y_{d p k j}\right\} & \geq 0, \text { for } \forall 1 \leq i, j \leq n, \\
\sum_{j=1}^{n}\left(\bar{a}_{p i j}+\bar{a}_{d p i j}\right) h_{j}+\sum_{j=1}^{n} \sum_{k=1}^{m} \max \left\{\underline{b}_{p i k}\left(y_{p k j}+y_{d p k j}\right),\right. & \\
\left.\bar{b}_{p i k}\left(y_{p k j}+y_{d p k j}\right)\right\} & <0, \text { for } \forall 1 \leq i \leq n, \\
h_{j} & \leq \bar{x}_{j}, \text { for } \forall 1 \leq j \leq n,
\end{aligned}
$$

then the closed-loop system (3) is not only positive but also asymptotically stable by a set of controllers $u(t)=F_{\sigma(t)} x(t)+F_{d \sigma(t)} x(t-d(t))$ for arbitrary switching signal. For $p \in \mathcal{P}, F_{p}$ and $F_{d p}$ are given by

$$
F_{p}=Y_{p} H^{-1}, F_{d p}=Y_{d p} H^{-1} .
$$

Furthermore, let $\bar{x}=\left[\bar{x}_{1}, \bar{x}_{2}, \cdots, \bar{x}_{n}\right]^{T}$, then $0 \preceq x(t) \preceq \bar{x}$ holds for $0 \preceq \varphi(t) \preceq h$.

Proof. It is easy to finish based on the proof of Theorem 5, so it is omitted.

\section{Synthesis with Polytopic Uncertainties}

In this section, we aim to extend the results in Section 3 to switched linear systems with polytopic uncertainties. Assume that the subsystem matrices belong to the uncertainty set

$$
\Theta_{p}:=\left\{\sum_{s=1}^{L} \gamma_{s}\left[A_{p}^{(s)}, A_{d p}^{(s)}, B_{p}^{(s)}\right] \mid \sum_{s=1}^{L} \gamma_{s}=1, \gamma_{s} \geq 0\right\}
$$


where $A_{p}^{(s)}, A_{d p}^{(s)}$ and $B_{p}^{(s)}$ are given matrices denoting the extreme points of the $p$-th subsystem for $s=1,2, \ldots, L$, and $L$ means the total number of extreme points.

\subsection{Sign-Restricted Control}

In this pat, the objective is to solve the robust stabilization issue of systems with polytopic uncertainties by nonnegative and negative controls.

Theorem 9. Consider system (1) with polytopic uncertainties, if there exist matrices $H=$ $\operatorname{diag}\left\{h_{1}, h_{2}, \ldots, h_{n}\right\} \succ 0, Y_{p}=\left[y_{p i j}\right] \succeq 0 \in \mathcal{R}^{m \times n}$ and $Y_{d p}=\left[y_{d p i j}\right] \succeq 0 \in \mathcal{R}^{m \times n}$ for $p \in \mathcal{P}$ such that

$$
\begin{aligned}
a_{p i j}^{(s)} h_{j}+\sum_{k=1}^{m} b_{p i k}^{(s)} y_{p k j} & \geq 0, \text { for } \forall 1 \leq i \neq j \leq n, \\
a_{d p i j}^{(s)} h_{j}+\sum_{k=1}^{m} b_{p i k}^{(s)} y_{d p k j} & \geq 0, \text { for } \forall 1 \leq i, j \leq n, \\
\sum_{j=1}^{n}\left(a_{p i j}^{(s)}+a_{d p i j}^{(s)}\right) h_{j}+\sum_{j=1}^{n} \sum_{k=1}^{m} b_{p i k}^{(s)}\left(y_{p k j}+y_{d p k j}\right) & <0, \text { for } \forall 1 \leq i \leq n,
\end{aligned}
$$

then the closed-loop system (3) is not only positive but also stable by a set of nonnegative controllers $u(t)=F_{\sigma(t)} x(t)+F_{d \sigma(t)} x(t-d(t))$ for arbitrary switching signal. For $p \in \mathcal{P}, F_{p}$ and $F_{d p}$ can be derived by

$$
F_{p}=Y_{p} H^{-1}, F_{d p}=Y_{d p} H^{-1}
$$

Proof. It is straightforward by a convexity argument from Theorem 1.

Corollary 5. Consider system (1), if there exist matrices $H=\operatorname{diag}\left\{h_{1}, h_{2}, \ldots, h_{n}\right\} \succ 0, Y_{p}=\left[y_{p i j}\right] \prec$ $0 \in \mathcal{R}^{m \times n}$ and $Y_{d p}=\left[y_{d p i j}\right] \prec 0 \in \mathcal{R}^{m \times n}$ for $p \in \mathcal{P}$ satisfying

$$
\begin{aligned}
a_{p i j}^{(s)} h_{j}+\sum_{k=1}^{m} b_{p i k}^{(s)} y_{p k j} & \geq 0, \text { for } \forall 1 \leq i \neq j \leq n, \\
a_{d p i j}^{(s)} h_{j}+\sum_{k=1}^{m} b_{p i k}^{(s)} y_{d p k j} & \geq 0, \text { for } \forall 1 \leq i, j \leq n, \\
\sum_{j=1}^{n}\left(a_{p i j}^{(s)}+a_{d p i j}^{(s)}\right) h_{j}+\sum_{j=1}^{n} \sum_{k=1}^{m} b_{p i k}^{(s)}\left(y_{p k j}+y_{d p k j}\right) & <0, \text { for } \forall 1 \leq i \leq n,
\end{aligned}
$$

then the closed-loop system (3) is positive and stable by a set of negative controllers $u(t)=F_{\sigma(t)} x(t)+$ $F_{d \sigma(t)} x(t-d(t))$ for arbitrary switching signal. For $p \in \mathcal{P}, F_{p}$ and $F_{d p}$ are given by

$$
F_{p}=Y_{p} H^{-1}, F_{d p}=Y_{d p} H^{-1}
$$

\subsection{Bounded and Sign-Restricted Controls}

This part considers controller synthesis issue under sign-restricted and bounded controls for systems with polytopic uncertainties. 
Theorem 10. Consider system (1) with polytopic uncertainties, if there exist matrices $H=$ $\operatorname{diag}\left\{h_{1}, h_{2}, \ldots, h_{n}\right\} \succ 0, Y_{p}=\left[y_{p i j}\right] \succeq 0 \in \mathcal{R}^{m \times n}$ and $Y_{d p}=\left[y_{d p i j}\right] \succeq 0 \in \mathcal{R}^{m \times n} p \in \mathcal{P}$ satisfying

$$
\begin{aligned}
a_{p i j}^{(s)} h_{j}+\sum_{k=1}^{m} b_{p i k}^{(s)} y_{p k j} & \geq 0, \text { for } \forall 1 \leq i \neq j \leq n, \\
a_{d p i j}^{(s)} h_{j}+\sum_{k=1}^{m} b_{p i k}^{(s)} y_{d p k j} & \geq 0, \text { for } \forall 1 \leq i, j \leq n, \\
\sum_{j=1}^{n}\left(a_{p i j}^{(s)}+a_{d p i j}^{(s)}\right) h_{j}+\sum_{j=1}^{n} \sum_{k=1}^{m} b_{p i k}^{(s)}\left(y_{p k j}+y_{d p k j}\right) & <0, \text { for } \forall 1 \leq i \leq n, \\
\sum_{j=1}^{n}\left(y_{p k j}+y_{d p k j}\right) & \leq \bar{u}_{k}, \text { for } \forall 1 \leq k \leq m,
\end{aligned}
$$

then the closed-loop system (3) is positive and asymptotically stable by a set of controllers $u(t)=$ $F_{\sigma(t)} x(t)+F_{d \sigma(t)} x(t-d(t))$ for arbitrary switching signal. For $p \in \mathcal{P}, F_{p}$ and $F_{d p}$ can be obtained by

$$
F_{p}=Y_{p} H^{-1}, F_{d p}=Y_{d p} H^{-1}
$$

For defined $\bar{u}=\left[\bar{u}_{1}, \bar{u}_{2}, \cdots, \bar{u}_{m}\right]^{T} \succ 0,0 \preceq u(t) \preceq \bar{u}$ sets up for any initial condition satisfying $0 \preceq x(0) \preceq h$.

Proof. It is straightforward by a convexity argument from Theorem 2.

Corollary 6. Consider system (1) with polytopic uncertainties, if there exist matrices $H=$ $\operatorname{diag}\left\{h_{1}, h_{2}, \ldots, h_{n}\right\} \succ 0, Y_{p}=\left[y_{p i j}\right] \prec 0 \in \mathcal{R}^{m \times n}$ and $Y_{d p}=\left[y_{\text {dpij }}\right] \prec 0 \in \mathcal{R}^{m \times n}$ for $p \in \mathcal{P}$ such that

$$
\begin{aligned}
& a_{p i j}^{(s)} h_{j}+\sum_{k=1}^{m} b_{p i k}^{(s)} y_{p k j} \geq 0, \text { for } \forall 1 \leq i \neq j \leq n, \\
& a_{d p i j}^{(s)} h_{j}+\sum_{k=1}^{m} b_{p i k}^{(s)} y_{d p k j} \geq 0, \text { for } \forall 1 \leq i, j \leq n, \\
& \sum_{j=1}^{n}\left(a_{p i j}^{(s)}+a_{d p i j}^{(s)}\right) h_{j}+\sum_{j=1}^{n} \sum_{k=1}^{m} b_{p i k}^{(s)}\left(y_{p k j}+y_{d p k j}\right)<0, \text { for } \forall 1 \leq i \leq n, \\
& \tilde{u}_{k} \leq \sum_{j=1}^{n}\left(y_{p k j}+y_{d p k j}\right), \text { for } \forall 1 \leq k \leq m,
\end{aligned}
$$

then the closed-loop system (3) is positive and asymptotically stable by a set of controllers $u(t)=$ $F_{\sigma(t)} x(t)+F_{d \sigma(t)} x(t-d(t))$ for arbitrary switching signal. For $p \in \mathcal{P}, F_{p}$ and $F_{d p}$ are given by

$$
F_{p}=Y_{p} H^{-1}, F_{d p}=Y_{d p} H^{-1}
$$

Moreover, define $\tilde{u}=\left[\tilde{u}_{1}, \tilde{u}_{2}, \cdots, \tilde{u}_{m}\right]^{T} \prec 0$, then $\tilde{u} \preceq u(t) \preceq 0$ is established for any initial condition satisfying $0 \preceq x(0) \preceq h$. 


\subsection{Asymmetrically Bounded Control}

In this part, we focus on the following problem: for prescribed $\bar{u}^{1} \succ 0$ and $\bar{u}^{2} \succ 0$, the aim is to determine input controller $-\bar{u}^{1} \preceq u(t) \preceq \bar{u}^{2}$ such that the resulting closed-loop system is positive and asymptotically stable for any initial condition $0 \preceq \varphi(t) \preceq h$.

Theorem 11. Consider system (1) with polytopic uncertainties, if there exist matrices $H=$ $\operatorname{diag}\left\{h_{1}, h_{2}, \ldots, h_{n}\right\} \succ 0, Y_{p}^{l}=\left[y_{p i j}^{l}\right] \succeq 0 \in \mathcal{R}^{m \times n}$ and $Y_{d p}^{l}=\left[y_{\text {dpij }}^{l}\right] \succeq 0 \in \mathcal{R}^{m \times n} p \in \mathcal{P}, l \in\{1,2$, subject to

$$
\begin{aligned}
a_{p i j}^{(s)} h_{j}+\sum_{k=1}^{m} b_{p i k}^{(s)}\left(y_{p k j}^{1}-y_{p k j}^{2}\right) & \geq 0, \text { for } \forall 1 \leq i \neq j \leq n, \\
a_{d p i j}^{(s)} h_{j}+\sum_{k=1}^{m} b_{p i k}^{(s)}\left(y_{d p k j}^{1}-y_{d p k j}^{2}\right) & \geq 0, \text { for } \forall 1 \leq i, j \leq n, \\
\sum_{j=1}^{n}\left(a_{p i j}^{(s)}+a_{d p i j}^{(s)}\right) h_{j}+\sum_{j=1}^{n} \sum_{k=1}^{m} b_{p i k}^{(s)}\left(y_{p k j}^{1}+y_{d p k j}^{1}-y_{l p k j}^{2}-y_{d p k j}^{2}\right) & <0, \text { for } \forall 1 \leq i \leq n, \\
\sum_{j=1}^{n}\left(y_{p k j}^{1}+y_{d p k j}^{1}\right) & \leq \bar{u}_{k}^{2}, \text { for } \forall 1 \leq k \leq m, \\
\sum_{j=1}^{n}\left(y_{p k j}^{2}+y_{d p k j}^{2}\right) & \leq \bar{u}_{k}^{1}, \text { for } \forall 1 \leq k \leq m,
\end{aligned}
$$

then the closed-loop system (3) is positive and asymptotically stable under a set of controllers with memory $u(t)=F_{\sigma(t)} x(t)+F_{d \sigma(t)} x(t-d(t))$. For $p \in \mathcal{P}, F_{p} F_{d p}$ are given by

$$
F_{p}=\left(Y_{p}^{1}-Y_{p}^{2}\right) H^{-1}, F_{d p}=\left(Y_{d p}^{1}-Y_{d p}^{2}\right) H^{-1} .
$$

Besides, choose $\bar{u}^{1}=\left[\bar{u}_{1}^{1}, \bar{u}_{2}^{1}, \cdots, \bar{u}_{m}^{1}\right]^{T}$ and $\bar{u}^{2}=\left[\bar{u}_{1}^{2}, \bar{u}_{2}^{2}, \cdots, \bar{u}_{m}^{2}\right]^{T}$, then $-\bar{u}^{1} \preceq u(t) \preceq \bar{u}^{2}$ sets up for any initial condition fulfilling $0 \preceq \varphi(t) \preceq h$.

Proof. It follows the proof of Theorem 3 because the convexity argument.

\subsection{Synthesis with State Constraints}

In this subsection, we consider the controller synthesis issue for systems with polytopic uncertainties. Our aim is to determine $u(t)$, such that the closed-loop system is asymptotically stable and the state is constrained in the box: $0 \preceq x \preceq \bar{x}$. 
Theorem 12. Consider system (1) with polytopic uncertainties, if there exist matrices $H=$ $\operatorname{diag}\left\{h_{1}, h_{2}, \ldots, h_{n}\right\} \succ 0, Y_{p}=\left[y_{p i j}\right] \in \mathcal{R}^{m \times n}$ and $Y_{d p}=\left[y_{d p i j}\right] \in \mathcal{R}^{m \times n} p \in \mathcal{P}$ such that

$$
\begin{aligned}
& a_{p i j}^{(s)} h_{j}+\sum_{k=1}^{m} b_{p i k}^{(s)} y_{p k j} \geq 0, \text { for } \forall 1 \leq i \neq j \leq n, \\
& a_{d p i j}^{(s)} h_{j}+\sum_{k=1}^{m} b_{p i k}^{(s)} y_{d p k j} \geq 0, \text { for } \forall 1 \leq i, j \leq n, \\
& \sum_{j=1}^{n}\left(a_{p i j}^{(s)}+a_{d p i j}^{(s)}\right) h_{j}+\sum_{j=1}^{n} \sum_{k=1}^{m} b_{p i k}^{(s)}\left(y_{p k j}+y_{d p k j}\right)<0, \text { for } \forall 1 \leq i \leq n, \\
& h_{j} \leq \bar{x}_{j}, \text { for } \forall 1 \leq j \leq n,
\end{aligned}
$$

then the closed-loop system (3) is not only positive but also asymptotically stable by a set of controllers $u(t)=F_{\sigma(t)} x(t)+F_{d \sigma(t)} x(t-d(t))$ for arbitrary switching signal. For $p \in \mathcal{P}, F_{p}$ and $F_{d p}$ are given by

$$
F_{p}=Y_{p} H^{-1}, F_{d p}=Y_{d p} H^{-1}
$$

Furthermore, select $\bar{x}=\left[\bar{x}_{1}, \bar{x}_{2}, \cdots, \bar{x}_{n}\right]^{T} \succ 0$, then $0 \preceq x(t) \preceq \bar{x}$ holds on for $0 \preceq \varphi(t) \preceq h$.

Proof. It is easy to finish according to the proof of Theorem 4, so it is omitted.

Remark 2. If the matrices $A_{p}, A_{d p}$ or $B_{p}$ are known, it is special situation of the results of Section 5. This is based on the fact $A_{p}^{(s)}=A_{p}, A_{d p}^{(s)}=A_{d p}$ or $B_{p}^{(s)}=B_{p}$ for $\forall 1 \leq s \leq L$. Moreover, it is the general issue when $A_{p}, A_{d p}$ and $B_{p}$ are all fixed.

Remark 3. In fact, direct application of Theorem 9 is cumbersome to compute due to the number of vertices might be very large. However, if $A_{p}^{(s)} \in\left[\underline{A}_{p}, \bar{A}_{p}\right], A_{d p}^{(s)} \in\left[\underline{A}_{d p}, \bar{A}_{d p}\right], B_{p}^{(s)} \in\left[\underline{B}_{p}, \bar{B}_{p}\right]$ for each $p \in \mathcal{P}, s \in\{1,2, \cdots, L\}$, thus we can cast the robust stabilization issue of system with polytopic uncertainties as system with interval uncertainties.

Remark 4. The results of time-varying delay can be also applied to the constant delay in this paper.

Remark 5. We use linprog function in Matlab to solve LP and get feasible solutions for LP problems.

\section{Numerical Example}

In this section, two examples are provided to illustrate the theoretical results.

Example 1. Consider system (1) with the following system matrices

$$
\begin{gathered}
A_{1}=\left(\begin{array}{cc}
-0.54 & -0.42 \\
0.18 & -0.61
\end{array}\right), A_{d 1}=\left(\begin{array}{cc}
-1.16 & -0.68 \\
0.2 & 0.4
\end{array}\right), B_{1}=\left(\begin{array}{l}
1.5 \\
0.2
\end{array}\right) \\
A_{2}=\left(\begin{array}{cc}
0.45 & 0.38 \\
-0.2 & -0.6
\end{array}\right), A_{d 2}=\left(\begin{array}{cc}
1.4 & 0.4 \\
-0.2 & 0.2
\end{array}\right), B_{2}=\left(\begin{array}{c}
-1.2 \\
0.4
\end{array}\right)
\end{gathered}
$$


It can be seen that all of the system matrices $A_{i}$ and $A_{d i}$ (for $\left.i=1,2\right)$ are not positive. The objective here is to design a set of constrained state-feedback controllers with memory $0 \preceq u(t) \preceq \bar{u}$ that stabilizes the system and enforces the state to be positive. Take $\bar{u}=100, d(t)=1+\sin (t)$. By Theorem 2, we can obtain a set of solutions by LP as $H=\operatorname{diag}\{11.6995,108.0623\}$ and

$$
\begin{aligned}
& Y_{1}=\left(\begin{array}{ll}
0.2251 & 31.0176
\end{array}\right), Y_{d 1}=\left(\begin{array}{ll}
10.0913 & 49.7589
\end{array}\right) \\
& Y_{2}=\left(\begin{array}{ll}
59.2217 & 12.1283
\end{array}\right), Y_{d 2}=\left(\begin{array}{ll}
9.6516 & 15.0621
\end{array}\right) .
\end{aligned}
$$

Then, according to (18), we can get

$$
\begin{aligned}
& F_{1}=\left(\begin{array}{ll}
0.0192 & 0.2870
\end{array}\right), F_{d 1}=\left(\begin{array}{ll}
0.8625 & 0.4605
\end{array}\right) ; \\
& F_{2}=\left(\begin{array}{ll}
5.0619 & 0.1122
\end{array}\right), \quad F_{d 2}=\left(\begin{array}{ll}
0.8250 & 0.1394
\end{array}\right) .
\end{aligned}
$$

By calculating, we have

$$
\begin{aligned}
& A_{1}+B_{1} F_{1}=\left(\begin{array}{cc}
-0.5111 & 0.0106 \\
0.1838 & -0.5526
\end{array}\right), A_{d 1}+B_{1} F_{d 1}=\left(\begin{array}{ll}
0.1338 & 0.0107 \\
0.3725 & 0.4921
\end{array}\right) \\
& A_{2}+B_{2} F_{2}=\left(\begin{array}{cc}
-5.6243 & 0.2453 \\
1.8248 & -0.5551
\end{array}\right), A_{d 2}+B_{2} F_{d 2}=\left(\begin{array}{ll}
0.4101 & 0.2327 \\
0.1300 & 0.2558
\end{array}\right) .
\end{aligned}
$$

Note that $A_{1}+B_{1} F_{1}, A_{2}+B_{2} F_{2}$ are Metzler matrices, and $A_{d 1}+B_{1} F_{d 1}, A_{d 2}+B_{2} F_{d 2}$ are positive matrices, thus the closed-loop system is positive.

The state evolution with initial conditions $\varphi(t)=(10,100)^{\top}$ are shown in Figure 1, it is clear to see that the systems are positive and stable. Figure 2 shows that the control input under the switching signal $\sigma(t)$. The arbitrary switching signal $\sigma(t)$ is described by Figure 3.

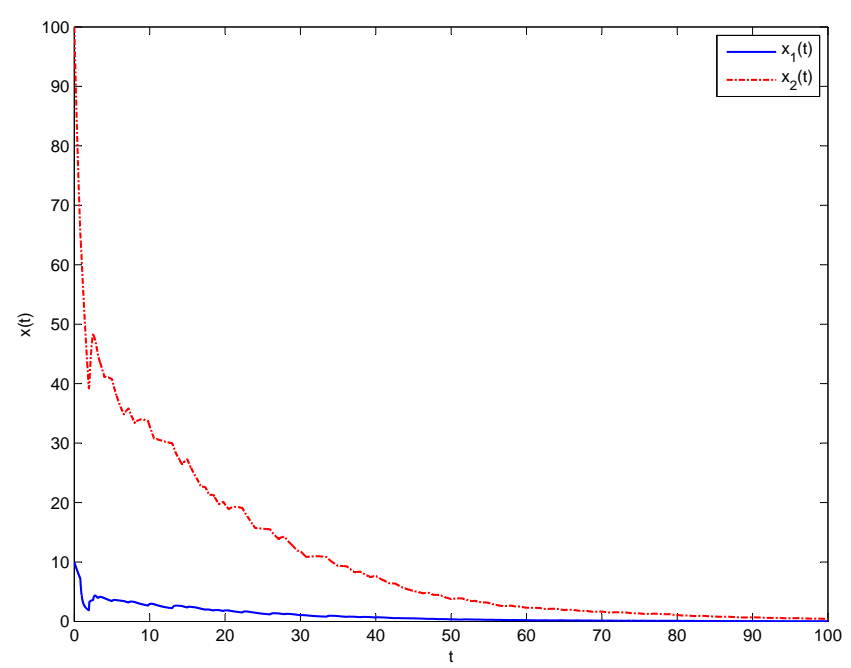

Figure 1. Closed-loop state response under the switching signal $\sigma(t)$ for Example 1. 


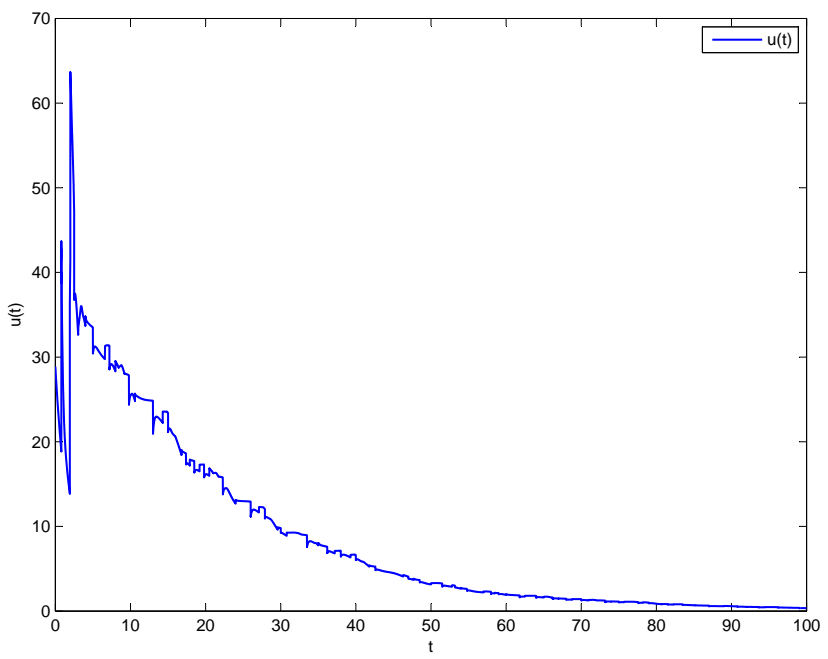

Figure 2. Control input under the switching signal $\sigma(t)$ for Example 1 .

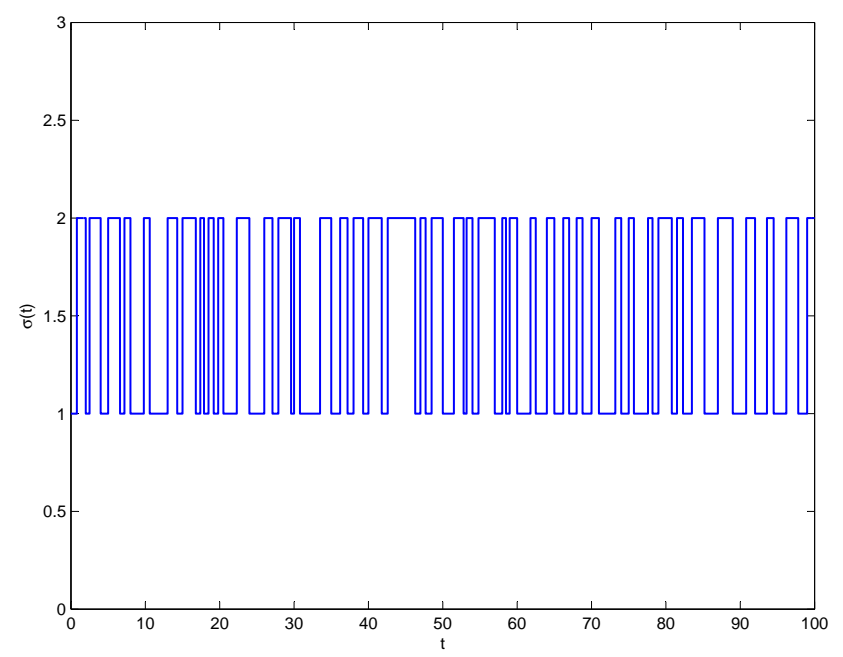

Figure 3. The switching signal $\sigma(t)$ for Example 1.

Example 2. Consider system (1) with the following system matrices

$$
\begin{gathered}
A_{1}=\left(\begin{array}{cc}
-2.5 & 1.3 \\
1.2 & -1.2
\end{array}\right), A_{d 1}=\left(\begin{array}{ll}
0.5 & 0.2 \\
0.3 & 0.2
\end{array}\right), B_{1}=\left(\begin{array}{ll}
0.1 & 0.2 \\
0.2 & 0.3
\end{array}\right) \\
A_{2}=\left(\begin{array}{cc}
-2 & 1.5 \\
0.5 & -1.2
\end{array}\right), A_{d 2}=\left(\begin{array}{ll}
0.2 & 0.5 \\
0.5 & 0.2
\end{array}\right), B_{2}=\left(\begin{array}{ll}
0.2 & 0.2 \\
0.3 & 0.3
\end{array}\right)
\end{gathered}
$$

It can be seen that the system matrices $A_{i}$ is Metzler matrix and $A_{d i}$ is positive (for $i=1,2$ ). The objective here is to design a set of constrained state-feedback controllers with memory $\tilde{u} \preceq u(t) \preceq 0$ that stabilizes the system and enforces the state to be positive. Choose $\tilde{u}=[-6,-8]^{T}, d(t)=0.5$. By Corollary 2, we can obtain a set of solutions by LP as $H=\operatorname{diag}\{3.4584,3.7627\}$ and

$$
Y_{1}=\left(\begin{array}{ll}
-1.2520 & -1.3556 \\
-1.8555 & -2.1020
\end{array}\right), \quad Y_{d 1}=\left(\begin{array}{ll}
-1.0997 & -1.0144 \\
-1.3803 & -1.1979
\end{array}\right)
$$




$$
Y_{2}=\left(\begin{array}{ll}
-1.4153 & -1.7416 \\
-1.9117 & -2.4676
\end{array}\right), Y_{d 2}=\left(\begin{array}{ll}
-1.1237 & -1.0811 \\
-1.4777 & -1.4209
\end{array}\right)
$$

Then, according to (23), we can get

$$
\begin{aligned}
& F_{1}=\left(\begin{array}{ll}
-0.3620 & -0.3603 \\
-0.5365 & -0.5586
\end{array}\right), \quad F_{d 1}=\left(\begin{array}{ll}
-0.3180 & -0.2696 \\
-0.3991 & -0.3184
\end{array}\right) ; \\
& F_{2}=\left(\begin{array}{ll}
-0.4092 & -0.4629 \\
-0.5528 & -0.6558
\end{array}\right), \quad F_{d 2}=\left(\begin{array}{ll}
-0.3249 & -0.2873 \\
-0.4273 & -0.3776
\end{array}\right) .
\end{aligned}
$$

By calculating, we have

$$
\begin{aligned}
& A_{1}+B_{1} F_{1}=\left(\begin{array}{cc}
-2.6435 & 1.1522 \\
0.9666 & -1.4396
\end{array}\right), A_{d 1}+B_{1} F_{d 1}=\left(\begin{array}{cc}
0.3884 & 0.1094 \\
0.1167 & 0.0506
\end{array}\right) ; \\
& A_{2}+B_{2} F_{2}=\left(\begin{array}{cc}
-2.1924 & 1.2763 \\
0.2114 & -1.5356
\end{array}\right), A_{d 2}+B_{2} F_{d 2}=\left(\begin{array}{cc}
0.0 .0496 & 0.3670 \\
0.2743 & 0.0005
\end{array}\right) .
\end{aligned}
$$

Note that $A_{1}+B_{1} F_{1}, A_{2}+B_{2} F_{2}$ are Metzler matrices, and $A_{d 1}+B_{1} F_{d 1}, A_{d 2}+B_{2} F_{d 2}$ are positive matrices, thus the closed-loop system is positive under this controller. Figure 4 shows the state response under initial conditions $\varphi(t)=(3.4,3.7)^{\top}$. The control input under the switching signal $\sigma(t)$ is given by Figure 5. Figure 6 depicts the arbitrary switching signal $\sigma(t)$.

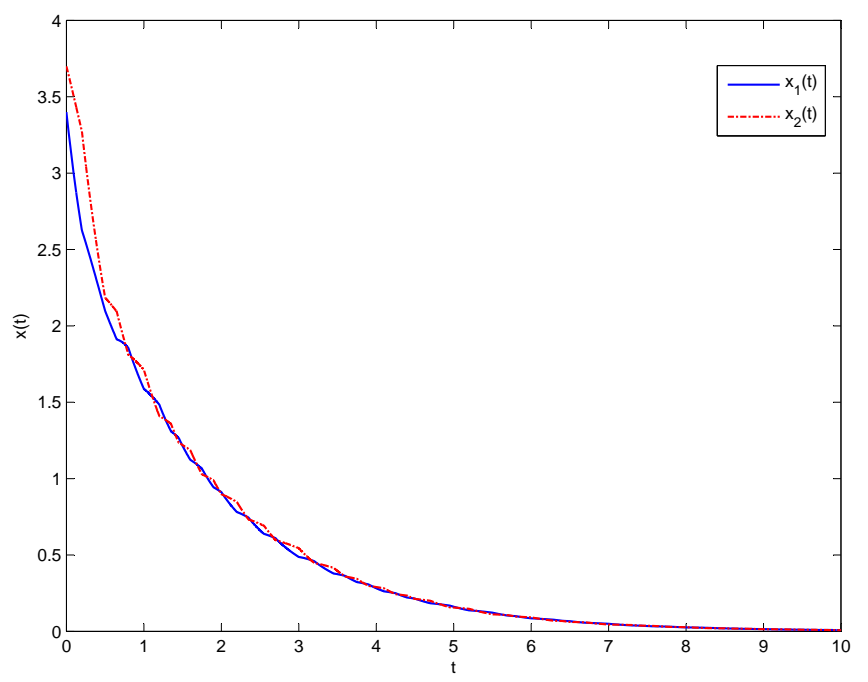

Figure 4. Closed-loop state response under the switching signal $\sigma(t)$ for Example 2. 


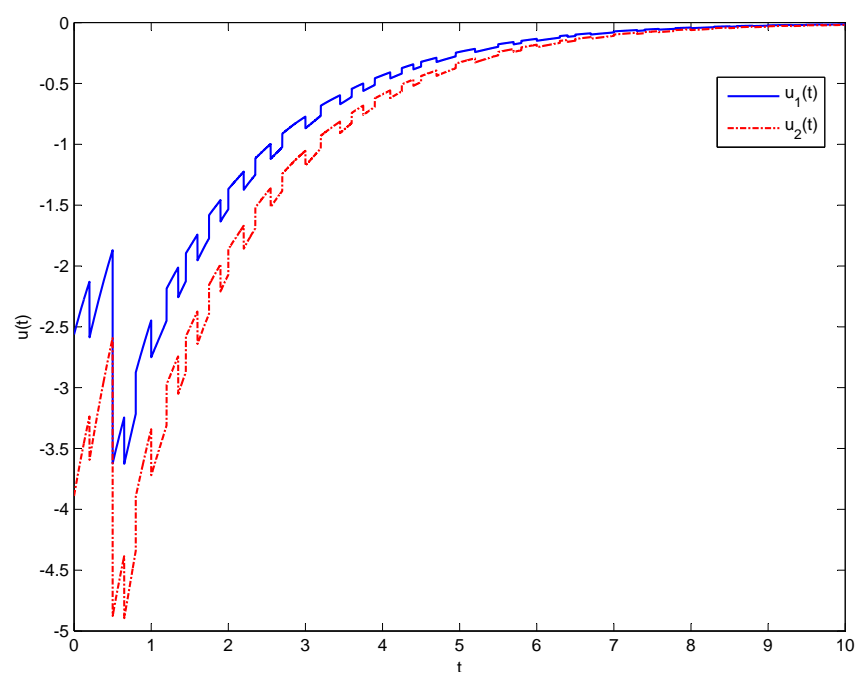

Figure 5. Control input under the switching signal $\sigma(t)$ for Example 2.

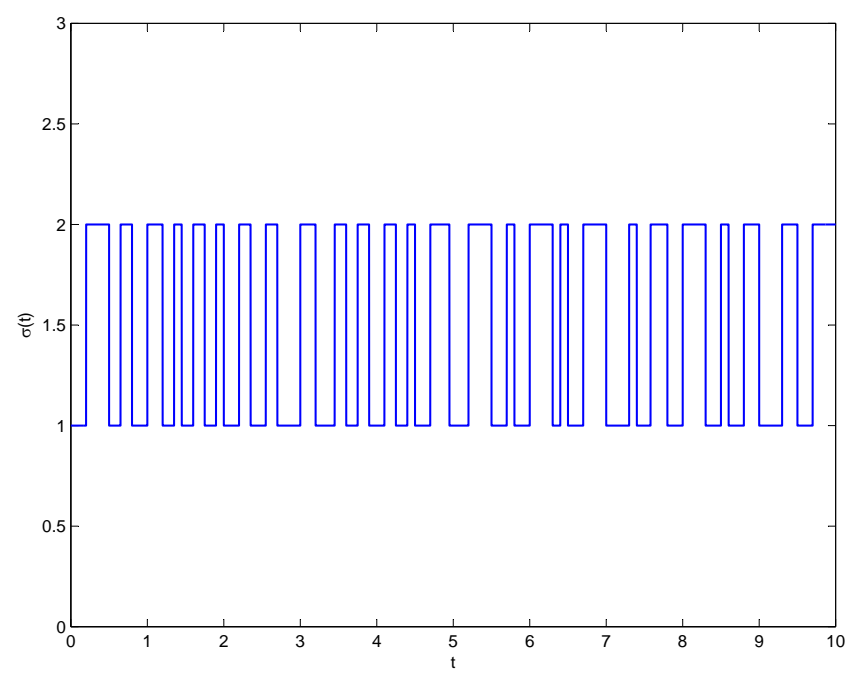

Figure 6. The switching signal $\sigma(t)$ for Example 2.

\section{Conclusions}

This paper has provided an approach for solving the stabilization issue for constrained switched linear systems with delay. The designed state-feedback with memory guarantees the positivity and stability of the closed-loop system. Moreover, the control and state are constrained by prescribed bound. The results are also extended to the systems with interval and polytopic uncertainties. It has been shown that all the derived sufficient conditions are solvable by simply LP. Following the approach we obtained in this paper, we further consider switched linear systems under average dwell time switching strategy.

\section{Acknowledgments}

The author would like to thank the editors and referees for their constructive comments and suggestions. This work is supported by the National Natural Science Foundation of China (61374006) and the Major Program of National Natural Science Foundation of China (11190015). 


\section{Author Contributions}

Kanjian Zhang managed the overall progress and gave some useful advise on this project. Jinjin Liu carried out the theoretical analysis and numerical calculations for the results. Both authors have read and approved the final manuscript.

\section{Conflicts of Interest}

The authors declare no conflict of interest.

\section{References}

1. Benzaouia, A.; Hmamed, A. Regulator problem of linear continuous-time systems with constrained control. IEEE Trans. Autom. Control 1993, 38, 1556-1560.

2. Blanchini, F. Set invariance in control. Automatica 1999, 35, 1747-1767.

3. Maciejowski, J.M. Predictive Control with Constraints; Prentice Hall: Upper Saddle River, NJ, USA, 2000.

4. Henrion, D.; Tarbouriech, S.; Kucera, V. Control of linear systems subject to input constraints: A polynomial approach. Automatica 2001, 36, 597-604.

5. Rami, M.A.; Tadeo, F.; Benzaouia, A. Control of constrained positive discrete systems. In Proceedings of the 2007 American Control Conference, New York, NY, USA, 9-13 July 2007; pp. 5851-5856.

6. Rami, M.A.; Tadeo, F. Controller synthesis for positive linear systems with bounded controls. IEEE Trans. Circuits Syst. II 2007, 54, 151-155.

7. Shorten, R.; Narendra, K. Necessary and sufficient conditions for the existence of a common quadratic Lyapunov function for two stable second order linear time-invariant systems. In Proceeding of the 1999 Amercian Control Conference, San Diego, CA, USA, 2-4 June 1999; pp. 1410-1414.

8. Shorten, R.; Narendra, K.; Mason, O. A result on common quadratic Lyapunov functions. IEEE Trans. Autom. Control 2003, 48, 110-113.

9. Decarlo, R.A.; Branicky, M.S.; Pettersson,S.; Lennartson, B.; Antsaklis, P.J. Perspectives and results on the stability and stabilizability of hybrid systems. Proc. IEEE 2000, 88, 1069-1082.

10. Michel, A.N. Recent trends in the stability analysis of hybrid dynamical systems. IEEE Trans. Circuits Syst. I 1999, 46, 120-134.

11. Liberzon, D.; Morse, A.S. Basic problems in stability and design of switched systems. IEEE Control Syst. Magzine 1999, 19, 59-70.

12. Lin, H.; Antsaklis, P.J. Stability and stabilizability of switching linear systems: A survey of recent results. IEEE Trans. Autom. Control 2009, 54, 308-322.

13. Farina, L.; Rinaldi, S. Positive Linear Systems: Theory and Applications; Wiley: New York, NY, USA, 2000.

14. Shorten, R.; Wirth, F.; Leith, D. A positive systems model of TCP-like congestion control: asymptotic results. IEEE/ACM Trans. Netw. 2006, 14, 616-629. 
15. Jadbabaie, A.; Lin, J.; Morse, A.S. Coordination of groups of mobile autonomous agents using nearest neighbour rules. IEEE Trans. Autom. Control 2003, 48, 988-1001.

16. Vargas, E.H.; Colaneri, P.; Middleton, R.; Blanchini, F. Discrete-time control for switched positive systems with application to mitigating viral escape. Int. J. Robust Nonlinear Control 2011, 21, 1093-1111.

17. Mason, O.; Shorten, R. On linear copositive Lyapunov functions and the stability of switched positive linear systems. IEEE Trans. Autom. Control 2007, 52, 1346-1349.

18. Fornasini, E.; Valcher, M.E. Linear copositive Lyapunov functions for continuous-time positive switched systems. IEEE Trans. Autom. Control 2010, 55, 1933-1937.

19. Tong, Y.; Zhang, L.; Shi, P.; Wang, C. A common linear copositive Lyapunov function for switched positive linear systems with commutable subsystems. Int. J. Syst. Sci. 2013, 44, 1994-2003.

20. Zhao, X.; Zhang, L.; Shi, P. Stability of a class of switched positive linear time-delay systems. Int. J. Robust Nonlinear Control 2013, 23, 578-589.

21. Zhao, X.; Zhang, L.; Shi, P.; Liu, M. Stability of switched positive linear systems with average dwell time switching. Automatica 2012, 48, 1132-1137.

22. Zhang, J.; Han, Z. Robust stabilization of switched positive linear systems with uncertainties. Int. J. Control Autom. Syst. 2013, 11, 41-47.

23. Fornasini, E.; Valcher, M.E. Stability and stabilizability criteria for discrete-time positive switched systems. IEEE Trans. Autom. Control 2012, 57, 1208-1221.

24. Xiang, M.; Xiang, Z. Stability, $L_{1}$-gain and control synthesis for positive switched systems with time-varying delay. Nonlinear Anal. Hybrid Syst. 2013, 9, 9-17.

25. Zhang, J.; Han, Z.; Zhu, F. $L_{1}$-gain analysis and control synthesis of positive switched systems. Int. J. Syst. Sci. 2013, doi:10.1080/00207721.2013.849773.

26. Chen, G.; Yang, Y. Finite-time stability of switched positive linear systems. Int. J. Robust Nonlinear Control 2014, 24, 179-190.

27. Rami, M.A. Stability and stabilization of positive systems with time varying delays. Lect. Notes Control Inf. Sci. 2009, 389, 205-215.

28. Rami, M.A.; Schonlein, M.; Jordan, J. Estimation of linear positive systems with unknown time-varying delays. Eur. J. Control 2013, 19, 179-187.

29. Liu, X.; Dang, C. Stability analysis of positive switched linear systems with delays. IEEE Trans. Autom. Control 2011, 56, 1684-1690.

30. Liu, X. Constraints control of positive systems with delays. IEEE Trans. Autom. Control 2009, 54, 1596-1600.

31. Liu, X.; Wang, L.; Yu W.; Zhong, S. Constraints control of positive discrete-time systems with delays. IEEE Trans. Circuits Syst. II 2008, 55, 193-197.

32. Hmamed, A.; Benzaouia, A.; Rami, M.A.; Tadeo, F. Memoryless control to drive states of delayed continuous-time systems within the nonnegative orthant. In Proceedings of the 17th World Congress the International Federation of Automatic Control, Seoul, Korea, 6-11 July 2008; pp. 3934-3939.

33. Bolajraf, M.; Tadeo, F.; Alvarez T.; Rami, M.A. State-feedback with memory for controlled positivity with application to congestion control. IET Control Theory Appl. 2010, 4, 2041-2048. 
34. Hmamed, A.; Rami, M.A.; Benzaouia, A.; Tadeo, F. Stabilization under constrained states and controls of positive systems with time delays. Eur. J. Control 2012, 18, 182-190.

35. Liu, X.; Dang, C. Stability analysis of positive switched linear systems with delays. IEEE Trans. Autom. Control 2011, 57, 1684-1690.

36. Shen, J.; Lam, J. Constrained Control of Switched Positive Systems with Discrete and Distributed Delays. In Proceedings of the 33rd Chinese Control Conference, Nanjing, China, 28-30 July 2014; pp. 6031-6036.

(c) 2015 by the authors; licensee MDPI, Basel, Switzerland. This article is an open access article distributed under the terms and conditions of the Creative Commons Attribution license (http://creativecommons.org/licenses/by/4.0/). 